\title{
Histomorphological Pattern of Splenectomy Specimens: A Five- Year Study in a Tertiary Teaching Hospital
}

\author{
Dr.Babina Sarangthem, $\mathrm{MD}^{1}$, Dr.Gayatri Devi Pukhrambam, $\mathrm{MD}^{2}$, \\ Dr. Sharmila Laishram, $\mathrm{MD}^{3}$, Dr.A.Barindra Sharma, $\mathrm{MD}^{4}$, \\ Dr.Kaushik Debnath, $\mathrm{MD}^{5}$ \\ ${ }^{1,2,3}$ Assisstant Professor of Pathology, ${ }^{4}$ Ex-Assisstant Professor of Pathology, at present, Associate Professor \\ of Immunohaematology \& Blood Transfusion, ${ }^{5}$ Professor \& Head of Pathology, Regional Institute of Medical \\ Sciences, Imphal, Manipur, India
}

\begin{abstract}
Spleen, being the largest organ of the mononuclear phagocytic system is involved in many systemic inflammations, generalized haematopoietic disorders as well as metabolic disorders. By its anatomic location where it is not protected by a bony cage,makes it prone to traumatic injury.Splenectomy is also one of the modalities of treatment in haematological disorders like thalassaemias which will help in minimizing the need, frequency and complications of repeated blood transfusions.On the other hand,post-splenectomy complications may be life-threatening with a mortality of 50\% to $80 \%$. Nowadays, attempts are made to save splenic functions by performing repair of laceration in injuries or by partial splenectomy. Fine needle aspiration cytology(FNAC) , a non-invasive diagnostic procedure may be helpful in some conditions without undergoing the invasive surgical approach.
\end{abstract}

Splenic pathology has not been much studied in this region.The retrospective study was taken up to highlight the histomorphological pattern of surgically removed splenectomy specimens and to correlate with with the clinical conditions.A total of 20 splenectomy specimens were received in the five-year period and the findings are highlighted.

Key words: Extramedullary haematopoiesis, Thalassaemia, Traumatic spleen, Splenectomy

\section{Introduction}

Spleen is the largest organ of the mononuclear phagocytic system and is involved in all systemic inflammations, generalized haematopoietic disorders as well as metabolic disturbances [1]. The location of the spleen in the abdomen where it is not protected by a bony cage makes it very prone to traumatic injury. The incidence of traumatic rupture of the spleen increases due to rise in automobile accidents [2,3]. The spleen is often involved in many haematological disorders because of its active role in immunosurveillance. Due to the geographical location, the thalassaemias which are a heterogeneous group of inherited disorders characterized by underproduction of globin chains are quite prevalent [4]. Frequent blood transfusions and splenectomy are the only options to improve life expectancy and quality of life in thalassaemics. Surgeons usually perform a splenectomy as it eliminates the extracorpuscular mechanism responsible for the accelerated destruction of normal donor red cells in the patient's circulation thus minimizing the need, frequency and complications of repeated blood transfusions [5]. The response to splenectomy in thalassaemia is variable from patient to patient, but it is usually satisfactory,and it is long-lasting [6].However, the benefits should outweigh the complications of splenectomy. Overwhelming infections may occur days or years after splenectomy resulting in a mortality of $50 \%$ to $80 \%$. Because of this, attempts are made to save splenic function in children by performing repair of the laceration or by partial splenectomy[7].

Many pathologists learn morphological changes of spleen from post-mortem specimens which are frequently autolysed and the normal histologic landmarks are obscured. Splenic pathology has not been much studied in this region. This study aims to highlight the histomorphological patterns in surgically resected splenectomy specimens received in our centre and to correlate with the clinical indications which may contribute in better understanding of pathological changes.

\section{Material And Methods}

This is a retrospective study carried out in the histopathology section of Pathology department, Regional Institute of Medical Sciences,Imphal,Manipur,India. Twenty consecutive splenectomy specimens received during the period from January 2007 to December 2011 were included in the study. The indications for the splenectomy and other available relevant clinical information were recorded. The weight, size, capsule and other changes recorded in the grossing sheet were analysed. All the specimens were fixed in $10 \%$ formol saline for 24-48 hours after sectioning by bread loafing technique and processed. Besides the routine 
Hematoxylin and Eosin stain, special stains like Perl's Prussian Blue stain and Giemsa stain were done whenever necessary. The histopathological slides were reviewed and the microscopic findings were studied and correlated with the clinical data. The results were compiled and analysed.

\section{Results}

A total of twenty splenectomy specimens were received during the study period. There were 11 males and 9 females and M:F was found to be 1.2:1. The age of the patients ranged from 6 years to 60 years and mean age was found to be 26.25 years(Table 1). The highest number of splenectomy cases was in the age group of 21-30 and 31-40 years with five cases each. The incidence declined after 60 years. The majority of cases with $45 \%$ (9 cases) of splenectomy were indicated for trauma following road traffic accident (RTA) and blunt injury, followed by thalassaemia with $40 \%$ ( 8 cases), one case each for immune thrombocytopenic purpura (ITP), autoimmune hemolytic anemia and staging of adenocarcinoma of stomach (fundus). Capsular rupture, laceration and haemorrhage were the main gross findings in traumatically ruptured spleen. The specimens received for haematological disorders showed increase in size and weight. The highest weight of 2860 grams was encountered in a 12 year old female with thalassaemia major. Microscopically, there were evidence of capsular disruption, perisplenitis, haemorrhage (infarction) and fibrosis in traumatic spleens (Fig.1). Sections from splenectomy specimen for thalassaemia showed thickened capsule, marked fibrosis with partial effacement of white pulp, marked reticuloendothelial cell hyperplasia and increased hemosiderin-laden macrophages. Extramedullary haematopoiesis was observed in one case of thalassaemia (Fig.2).Splenic sections from ITP showed moderate reticuloendothelial cell hyperplasia. Sections from the spleen removed for autoimmune haemolytic anemia showed features consistent with hypersplenism. No case of malignancy was found in our study.

The splenic sections were also reviewed for any infectious pathology, particularly malaria and were found to be negative.

\section{Discussion}

Histopathological studies on the spleen are not common in this region. This study reports the histomorphological findings in twenty splenectomy specimens received during the five year study period. Seventy five percent of the splenectomies were found to be done in the $2^{\text {nd }}$ and $3^{\text {rd }}$ decade of life. This finding is comparable with the study of Al-Kindi $\mathrm{H}$ et al [3]. The higher incidence in this age group may be related to greater predisposition to traumatic injury in this age group. The age and gender distribution are close to the reported series in the literature $[3,8,9]$. The most common histopathological findings in our study were haemorrhagic necrosis and capsular disruption as a result of splenic laceration in road traffic accidents and blunt trauma. The higher frequency of splenic damage in road traffic accidents and blunt trauma to the abdomen have also been found from the studies of Smith J et al [10] and SayersRD et al [11]. The fact that the spleen is largely unprotected by the the ribs particularly when it enlarges also makes it more susceptible to damage. The other predisposing factors which could have contributed to the rupture were also looked into. In the present study, two specimens of traumatic rupture showed increased blackish brown pigments mimicking malarial pigments but definite malarial parasite could not be detected. In other studies predisposing factors like malaria, amyloidosis, follicular hyperplasia etc were detected in cases of ruptured spleen[3,8,9].

Out of the splenectomy specimens received for hematological disorders, $40 \%$ were for management of thalassaemia major. The increased number of these cases might be due to the fact that this region belongs to the thalassemic belt and the treatment option of splenectomy being safe and beneficial to these patients as studied by Babu TY et al [12].In the past splenectomy played a major role in the treatment of thalassaemia. Nowadays several alternatives have been proposed, the procedure most often reported and more successful has been partial embolization of the splenic artery[13].

The clinical manifestations of hypersplenism and iron overload were correlated with the histological evidence of thickened capsule, sinus histiocytic hyperplasia, paucity of white pulp and increased hemosiderin pigments as evidenced from the Perl's stained section.

Extramedullary haematopoiesis is a reactive condition which was observed in one case of spleen removed for management of thalassaemia. The hypoxia due to the congestion and ineffective circulating mature blood elements stimulate extramedullary haematopoiesis. This reactive condition may be confused with malignancy.[9].

The findings of reticulo-endothelial hyperplasia in a case of ITP may be compared with the study of Khalid A et al [9] . They observed that prominence of histiocytes in red pulp ( littoral cell hyperplasia ) are the result of phagocytosis of antibody coated platelets and incompletely degraded membrane derived phospholipids. Another study reported that in ITP, patients whose spleens exhibit neither prominent secondary reactive follicular hyperplasia nor ceroid histiocytosis have a significantly poorer response to splenectomy, leading to the conclusion that the thrombocytopenia in such patients may be from a different mechanism of 
action.[14].The splenectomy done for one case of adenocarcinoma ( fundal growth of stomach) in our study was for staging and no metastatic deposit or local spread was detected. The findings in relation to the clinical conditions will contribute in the study of splenic pathology.

Table 1: Age distribution and disease pattern

\begin{tabular}{|l|c|c|c|c|c|}
\hline $\begin{array}{c}\text { Age } \\
\text { range }\end{array}$ & Traumatic rupture & Thalassaemia & I T P & $\begin{array}{c}\text { AIHA } \\
\text { staging }\end{array}$ \\
\hline $0-10$ & 0 & 3 & & & \\
\hline $11-20$ & 2 & 1 & & & \\
\hline $21-30$ & 3 & 2 & & & \\
\hline $31-40$ & 2 & 1 & 1 & & \\
\hline $41-50$ & 1 & 1 & & & \\
\hline $51-60$ & 1 & 0 & 8 & 1 & 1 \\
\hline Total & 9 & 8 & & 1 \\
\hline
\end{tabular}

ITP: Idiopathic thrombocytopaenic purpura ; AIHA: Auto-immune haemolytic anemia

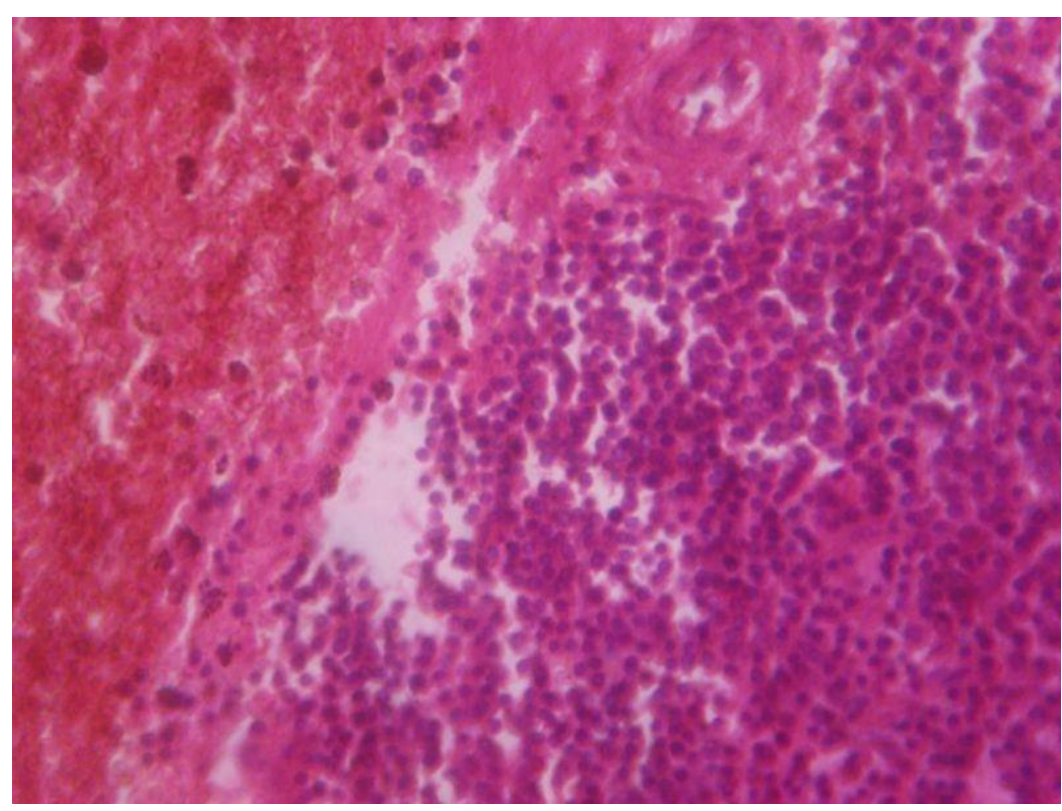

Figure 1. Microphotograph of traumatic spleen showing haemorrhagic necrosis and inflammation (perisplenitis) ( $\mathrm{H} \& \mathrm{E}, 40 \mathrm{x})$

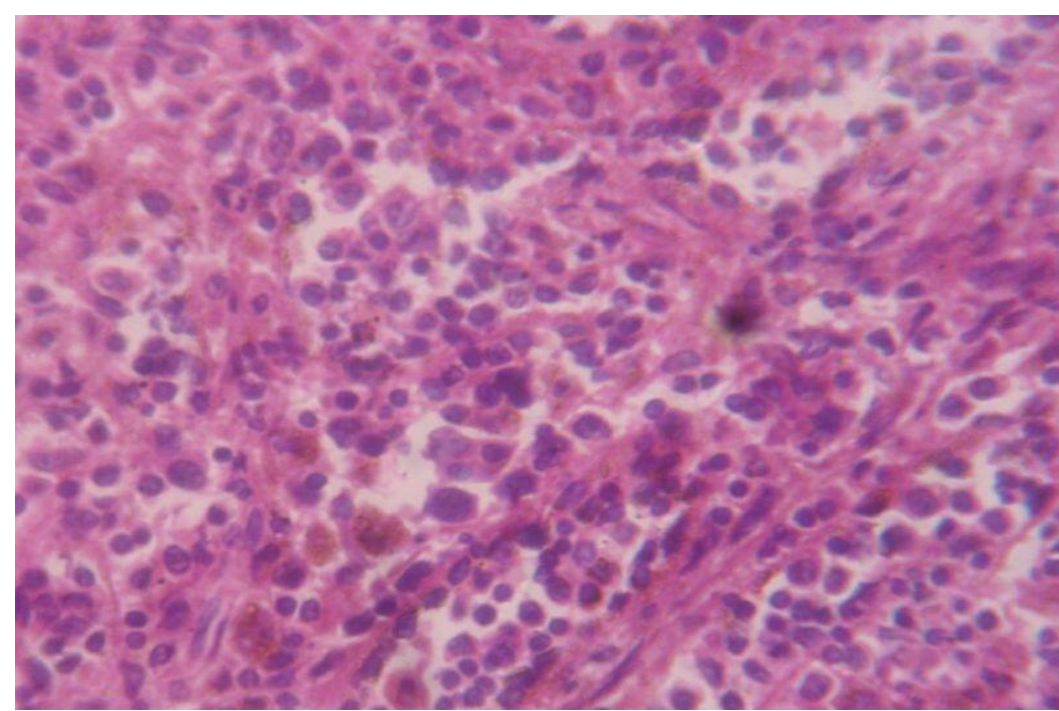

Figure 2. Microphotograph of extramedullary haematopoiesis showing precursor haemopoietic cells $(\mathrm{H} \& \mathrm{E}$, 40x) 


\section{Conclusion}

In this five year retrospective study of splenectomy specimens, majority of the cases showed features of traumatic pathology followed by histomorphological features related to haematological disorders predominantly thalassemia and one specimen sent for tumour staging showed normal histology. For a systematic approach to morphologic diagnosis, we need to pay more attention to the microscopic examination of white and red pulp.

In this study we have felt that in some of the cases, fine needle aspiration cytology (FNAC) could have been done as a noninvasive diagnostic procedure without undergoing the more aggressive surgical procedure. Other treatment options like partial splenectomy or repair of laceration may also be considered to avoid postsplenectomy complications. Reduction of RTA may proportionately reduce emergency splenectomies. Larger studies are needed for better understanding of this important organ and unnecessary splenectomies may thus be avoided.

\section{References}

[1]. Aster J C. Diseases of white blood cells, lymph nodes, spleen and thymus. In: Kumar V, Abbas AK, Fausto M. (Eds) Robbins and Cotran Pathologic basis of disease. Elsevier Saunders, Philadelphia, $7^{\text {th }}$ Ed. 2005: Pp702- 705 .

[2]. Ameh E A, Chirdan L B, Nmadu PT . Blunt abdominal trauma in children : epidemiology, management and management problems in a developing country. Paediatric Surgery International. 2000; 16: 505-509.

[3]. AL-Kindi H, Devi L, George M. Splenic pathology in traumatic rupture of the spleen: A five year study. Oman Medical Journal 2009; 24(2):81-83

[4]. Achoubi N, Asghar M, Saraswathy KN, Murry B. Prevalence of b-thalassemia and hemoglobin E in two migrant populations of Manipur, North East India. Genet Test Mol Biomarkers 2012; 16(10): 1195-1200

[5]. Al- Salem A H, Naserulla Z, Qaisaruddin S, Al-Dabbous I, Abkari H A, Al-Jama A et al . Splenectomy in hematological Diseases. Annals of Saudi Medicine 1999; $19: 325-330$.

[6]. Cohen A,Gayer R,Mizanin J. Long-term effect of splenectomy on transfusion requirements in thalassemia major. Am J Hematol 1989; 30: 254

[7]. Sherman R. Management of trauma to the spleen. Adv Surg 1984; 17: 37-71

[8]. Adelusola K A, Osasan S A, Afolabi O A. Histopathological study and audit of the spleen in Nigerians. Afr J Health Sci 2007; 14(3-4): 195-200.

[9]. Khalid A, U1 Haque A, Naseem L. Spectrum of disease entities in splenectomy specimen. International Journal of Pathology 2006; 4(2):88-93

[10]. Smith J, Caldwell E, D' Amours S, Jalaludin B, Surge M. Abdominal trauma: a disease in evolution. ANZ Journal of Surgery. 2005:75:790-794.

[11]. Sayers RD, Bewes PC, Porter KM. Emergency laparotomy for abdominal trauma. Injury 1992; 23:537-541.

[12]. Babu T Y, Kumar R, Singh O, Laishram J, Singh Th H. Splenectomy in thalassemia. Journal of Medical Society 2006; 20(2):9294.

[13]. Pringle KC, Spigos DG, Tan WS, Politis C, Pang EJ, Reyez HM. Partial splenic embolization in the management of thalassemia major. J Pediatr Surg 1982; 17: 884-91

[14]. Chang CS, Li CL,Cha SS. Chronic idiopathic thrombocytopaenic purpura: splenic pathologic features and their clinical correlations. Arch Pathol Lab Med 1993; 117: 981-985 